\title{
Perspectives in surgical ablation of atrial fibrillation
}

\section{Timo Weimar}

\author{
Sana Cardiac Surgery Stuttgart GmbH, Stuttgart, Germany
}

Despite the good results that can be achieved by the surgical treatment of atrial fibrillation (AF), still only a considerable small portion of patients presenting with this arrhythmia receive a concomitant ablation during their cardiac procedures and such remain exposed to their increased risk of stroke and hemodynamic compromises. While some surgeons still express the feeling that available evidence on the benefit of AF ablation is still lacking, the STS-guideline committee just recently reviewed the available literature and issued a la-Indication for adding an ablation during mitral valve but also aortic valve and coronary artery bypass graft procedures, clearly stating that the benefit exceeds the potential added risk by far.

Looking at the published literature we see a myriad of lesion sets and follow-up patterns applied, which not only dilute potential success rates but also make it nearly impossible to compare results. It is therefore critical to understand the Maze concept in order to achieve the best possible success rates and to anticipate the consequences when we decide to deviate from it, the working mode but also the limitations of the energy sources and devices we use and finally the need of getting appropriate training.

The lesion set strategy should be based on the underlying pathology. While in patients with paroxysmal AF, a pulmonary vein isolation (PVI) will isolate most of the triggers known to induce $A F$ and such is a reasonable approach for those patients, this strategy will not be sufficient in patients with non-paroxysmal AF, in which we additionally need to interrupt the macro-reentries sustaining the arrhythmia by an extended lesion set.

The biatrial Maze procedure remains the gold standard in terms of achievable success rates. This concept isolates the potential triggers and also interrupts all known macroreentries. Failing to isolate the entire posterior atrial wall - by leaving gaps or by omitting either the superior or inferior connecting lesion between the PVIs - will lead to a significant decrease in success. Further, the coronary sinus as one of the pathways for atypical atrial flutter needs to get special attention. This structure should be ablated endocardially with the so called mitral isthmus line going from the left PVI to the mitral annulus and also epicardially, making sure the lesions overlay each other.

It still is common practice for many surgeons to only address the left atrium. There are two large meta-analyses available comparing a left- to a bi-atrial lesion set. Ad et al. published a $10 \%$ better success rate for the bi-atrial lesion set, while Phang et al. could reveal this superiority only for the first year. However, we still have no tool yet to identify those patients who would benefit from the right-sided ablation. While it seems to be reasonable to address the left side only if there is no right-sided pathology such an enlarged atrium, tricuspid valve regurgitation, pulmonary hypertension etc., we should be aware of the fact that this might affect our success rate.

This balance also reflects our attitude toward AF. Stroke is a devastating consequence for patients. Still we consider AF minor compared to other cardiac diseases and are more concerned about adding risk to the procedure by an ablation. However, a recent analysis of the STS-Database by Rankin et al. revealed the omission of an ablation in patients with AF receiving double or triple valve surgery as one of two predictors for postoperative mortality. This in some kind repeats history in cardiac surgery. While in early days it was considered too dangerous to address the aortic valve stenosis and the coronary heart disease at the same time, we learned later that survival increased significantly when both problems were fixed within the same procedure. Even in the above mentioned patient group of double and triple valve surgery,

Address: Timo Weimar MD, Sana Cardiac Surgery Stuttgart GmbH, Herdweg 2, 70174 Stuttgart, Germany, e-mail: timo.weimar@sana.de (C) 2017, ČKS. Published by Elsevier sp. z 0.0. All rights reserved.

DOI: $10.1016 /$ j.crvasa.2017.06.013 
which I would consider high risk, the addition of an ablation did not increase perioperative complications but even improved early mortality.

The variability in atrial wall thickness and the degree of epicardial fat are some of the challenges current ablation devices face. To create reliable transmural lesions we have two energy sources available, which both have proofed to be safe and efficacious: Cryo- and Radiofrequency energy.

At $-20^{\circ} \mathrm{C}$, extracellular ice formation causes a hyperosmolar environment that leads to cell shrinking and finally cell death. Rapid freezing to $-40^{\circ} \mathrm{C}$ causes intracellular ice formation that destroys cell organelles and membranes even before osmotic imbalance occurs. The current ablation devices achieve those two markers. In addition, device specific factors as cooling agent, heat capacity and heat conduction coefficient also play a role for the efficacy of heat removal. We should ensure a perfect probe-tissue-interface without any faults to avoid ice ball formation that would work as isolator and might cause gaps. Further it is essential to overlap our lines of ablation since cryo probes tend to have their weakest performance at the very distal end. Cryo energy preserves collagen tissue and is therefore the energy of choice in the vicinity of the mitral and tricuspid valve. The heat sink effect will limit the efficacy of cryo energy if applied epicardially on the beating heart, so this cannot be recommended. However, the endocardial application for 2-3 min depending on the device/technology used will create reliable lines of transmurality in nearly $99 \%$.
Radiofrequency uses alternating current in the range of 100-1000 kilohertz $(\mathrm{kHz})$. The lesion size created by thermal injury depends on electrode-tissue contact area, the interface temperature, the current and voltage (power), as well as the duration of delivery. On histologic evaluation of radiofrequency lesions, a focal coagulation necrosis predominates acutely. This correlates with the irreversible nature of the injury, which occurs at temperatures $>50^{\circ} \mathrm{C}$. There is destruction of the myocardial collagen matrix and replacement with fibrin and collagen in chronic studies. At very high temperatures greater than $100{ }^{\circ} \mathrm{C}$, char formation predominates. Char presents as an impediment to heat transduction and has been associated with asymmetrical ablations. The depth of the lesion can be limited by char formation, epicardial fat, myocardial and endocavity blood flow, and tissue thickness. To avoid unintentional injury of adjacent tissue, it is my strong believe that only bipolar radiofrequency devices should be used nowadays due to their efficacy and safety.

Finally, a thought through training track for AF ablation is necessary to increase the success and adoption. So far this has not been integrated in most of our residence training programs. The lack of exposure to this field of cardiac surgery might have further contributed the vast variety of results reported. With the recent Guideline recommendation it is the surgeon's responsibility not only to perform some kind of ablation, but to give the patient the best possible chance for a better quality of life after the procedure. 